OPEN ACCESS

Edited by:

Sang-Hak Lee

Yonsei University, South Korea

Reviewed by:

Tetsuro Miyazaki,

Juntendo University Urayasu

Hospital, Japan

Seung-Jun Lee,

Severance Cardiovascular Hospital,

South Korea

${ }^{*}$ Correspondence:

Ran Gao

ggrr1991@outlook.com

Jing Wang

wangjing@ibms.pumc.edu.cn

Specialty section:

This article was submitted to

Cardiovascular Metabolism,

a section of the journal

Frontiers in Cardiovascular Medicine

Received: 17 November 2020

Accepted: 05 January 2021

Published: 05 February 2021

Citation:

Hou Y, Guo W, Fan T, Li B, Ge W, Gao $R$ and Wang J (2021) Advanced

Research of Abdominal Aortic

Aneurysms on Metabolism

Front. Cardiovasc. Med. 8:630269.

doi: 10.3389/fcvm.2021.630269

\section{Advanced Research of Abdominal Aortic Aneurysms on Metabolism}

\author{
Yangfeng Hou, Wenjun Guo, Tianfei Fan, Bolun Li, Weipeng Ge, Ran Gao* and Jing Wang \\ State Key Laboratory of Medical Molecular Biology, Institute of Basic Medicine, Chinese Academy of Medical Sciences, \\ School of Basic Medicine, Peking Union Medical College, Beijing, China
}

Abdominal aortic aneurysm (AAA) is a cardiovascular disease with a high risk of death, seriously threatening the life and health of people. The specific pathogenesis of AAA is still not fully understood. In recent years, researchers have found that amino acid, lipid, and carbohydrate metabolism disorders play important roles in the occurrence and development of AAA. This review is aimed to summarize the latest research progress of the relationship between AAA progression and body metabolism. The body metabolism is closely related to the occurrence and development of AAA. It is necessary to further investigate the pathogenesis of AAA from the perspective of metabolism to provide theoretical basis for AAA diagnosis and drug development.

Keywords: abdominal aortic aneurysm, metabolism, diabetes mellitus, hyperlipidemias, pathogenesis

\section{INTRODUCTION}

Abdominal aortic aneurysm (AAA) is a disease in which the abdominal aorta gradually expands like a tumor under the action of blood pressure. Usually, when the diameter of the abdominal aorta exceeds $3 \mathrm{~cm}$ or increases by more than $50 \%$ compared with normal, it can be diagnosed with AAA (1). AAA commonly occurs in elderly male over age of 65 , and the morbidity can reach $8 \%$ (1). The most serious consequence is the rupture of the artery wall due to it cannot withstand the impact of blood flow. The mortality from ruptured aneurysms can exceed $80 \%(1,2)$, and the mortality of patients undergoing repair surgery still exceeds $50 \%(1,3,4)$.

AAA is caused by the destruction of the abdominal aorta, especially the elastin break, due to a variety of congenital or acquired factors. Previous studies have found that the pathological process of AAA mainly includes local inflammatory cell infiltration, protease hydrolysis of elastic fibers and collagen fibers, vascular smooth muscle cell (VSMC) apoptosis and phenotypic transformation, and oxidative stress caused by oxidation and anti-oxidation imbalance $(1,2,5)$. In recent years, researchers have discovered that the metabolic disorders of amino acids, lipids and glucose in vivo are closely related to the occurrence and development of AAA by affecting the above-mentioned pathological processes. This article will review the latest research of the relationship between AAA and the metabolism of main nutrients in vivo.

\section{AMINO ACID METABOLISM AND AAA \\ Homocysteine}

Homocysteine (Hcy), an intermediate product in the metabolism of methionine and cysteine, is a sulfur-containing amino acid. Lack of key enzymes required for Hcy metabolism, such as cystathionine $\beta$-synthase (CBS), and coenzymes, such as folic acid, vitamin B6, and vitamin B12, will cause hyperhomocysteinemia (HHcy). 
HHcy is a risk factor for many cardiovascular diseases, including AAA (6-8). Early clinical studies found that HHcy caused by congenital CBS deficiency would increase the risk of vascular diseases, such as abdominal aortic aneurysm, pulmonary embolism, and myocardial infarction, and lowering of circulating Hcy level by long-term treatment can significantly improve the vascular outcome of patients with $\operatorname{CBS}$ deficiency $(9,10)$. One of the most representative drugs treating HHcy and entering phase $1 / 2$ clinical trials is OT-58, which is obtained from human CBS (11). Some preclinical studies provide evidence that OT-58 may be further investigated in AAA treatment in the future $(12,13)$.

HHcy may also increase the concentration and activity of MMP-9 in the blood vessel wall by activating ERK and Akt signaling pathways to promote the occurrence and development of AAA (14, 15). Siennicka et al. (16) found that high concentrations of $\mathrm{Hcy}$ can participate in the pathological process of AAA by affecting proteolysis and coagulation/fibrinolysis system. In AAA with thin intraluminal thrombus, high Hcy can increase matrix metalloproteinase (MMP)-2 and fibrinolytic factors (plasminogen and tissue-type plasminogen activator), leading to the degradation of elastin and collagen. On the contrary, in AAA with thicker thrombus, high level of Hcy produce the opposite effect, which may be one of reasons that lesion with thicker thrombus is less likely to rupture, and intraluminal thrombus may have some influence on the effect of HHcy on AAA (16). Besides, HHcy can also cause local inflammatory cell infiltration in AAA, increase the expression of inflammatory factors (such as IL-6), and promote the transformation of VSMC phenotype from contractile to synthetic (17). Moreover, HHcy can induce autophagy in VSMC by activating the AMPK signaling pathway, which is characterized by the increased expression of autophagy-related proteins LC3 and Beclin-1 (Figure 1a) (17).

Researchers have confirmed that serum Hcy level is positively correlated with the size, diameter, expansion rate, and risk of rupture of aneurysms in AAA patients (18-22). Therefore, some researchers have tried to inhibit the occurrence and development of AAA by regulating the metabolism of Hcy. Fan et al. (23) found that excessive supplementation of Hcy's metabolic precursor methionine can induce Hcy production and cause hypermethioninemia (HMET), thereby enhancing the expression of MMP-2 and inflammatory response in the vessel wall and exacerbating the development of AAA in rats. This suggests that for AAA patients, restricting the intake of methionine may have a protective effect. There is also preliminary evidence in clinical practice that the low-methionine diet combined with vitamin B6, vitamin $\mathrm{B} 12$ and folic acid can reduce the concentration of serum Hcy for HHcy patients with AAA, and may protect people from AAA $(10,24)$.

\section{Tryptophan}

Tryptophan (Trp), one of the essential amino acids, is catalyzed by indoleamine 2,3-dioxygenase (IDO) or tryptophan 2,3-dioxygenase (TDO) to generate kynurenine (Kyn). The

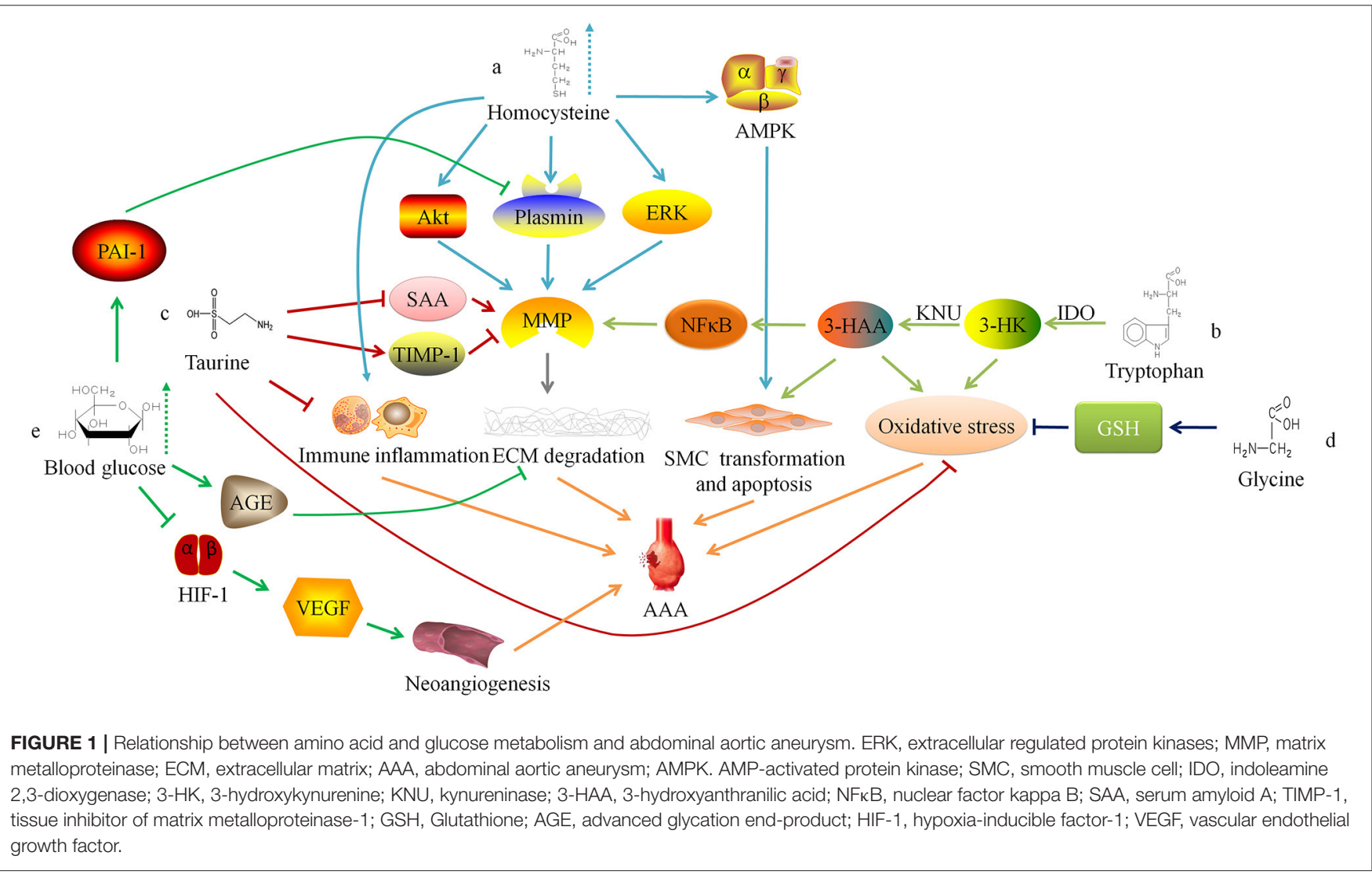


catabolism of Kyn can produce 3-hydroxykynurenine (3HK) and 3-hydroxyanthranilic acid (3-HAA). 3-HAA can be produced from $3-\mathrm{HK}$ under the action of kynureninase (KNU).

Wang et al. (25) found that the degradation of the elastic laminae and arterial expansion rate in $\mathrm{Apoe}^{-/-} \mathrm{IDO}^{-/-}$mice was significantly reduced. Further exploration of the mechanism found that 3-HAA up-regulated the expression of MMP-2 by activating the transcription factor nuclear factor kappa $\mathrm{B}$ $(\mathrm{NF} \kappa \mathrm{B})$. Therefore, knockdown of KNU in mice can inhibit the production of 3-HAA and MMP-2, thereby inhibiting the formation of AAA. The detection of human AAA tissue samples also found that the anti-3-HAA, anti-IDO and antiKNU antibody staining intensity in aneurysm tissue was stronger than that in non-aneurysm tissue sections (25). These results suggest that reducing the serum 3-HAA level in AAA patients by regulating Trp metabolism may be a potential therapeutic target. Furthermore, 3-HK and 3-HAA can generate free radicals (26), which may lead to an imbalance between oxidation and antioxidation in the blood vessel wall and promote AAA. Metghalchi et al. (27) found that IDO deficiency may limit the development of aneurysms by reducing the production of 3-HAA, but the specific mechanism needs further research (Figure 1b). At present, several IDO inhibitors, such as Indoximod, Epacadostat, and Navoximod, have been approved for patients in anti-tumor therapy, but there is a lack of research to assess the application of these drugs on AAA (28).

\section{Taurine}

Taurine (Tau) is an amino acid converted from sulfur-containing amino acids. Kim et al. (29) found that oral supplementation of Tau could inhibit AAA formation in mice, because Tau can react with the oxidant (such as $\mathrm{HOCl}$ ) catalyzed by myeloperoxidase to alleviate oxidative stress, reduce inflammatory cell aggregation, and inhibit activity of matrix metalloproteinase- 1 in the vessel wall. In addition, Tau can also reduce the serum amyloid A level (29), which can promote the AngII-induced formation of AAA in mice (30). These findings suggest that supplementing patients with Tau may be a potential prevention or treatment method for AAA (Figure 1c).

Besides, Tau can also bind with ursodeoxycholic acid to form tauroursodeoxycholic acid (TUDCA). By animal experiments, researchers found that TUDCA can reduce the apoptosis of VSMC by inhibiting endoplasmic reticulum stress, thereby reducing the maximum diameter of aneurysms induced by AngII (31).

\section{Glycine}

Glycine (Gly) can form the endogenous antioxidant glutathione (GSH) with glutamic acid and cysteine. Studies have found that Gly has protective effects on cardiovascular diseases, such as antagonizing cardiac and cerebral ischemic damage (32, $33)$, and lowering blood pressure $(34,35)$. The researchers found that supplementing the diet with an appropriate dose of Gly could prevent cardiovascular diseases $(36,37)$, which may depend on potential mechanisms showing below: (1) Activating the glycine-gated $\mathrm{Cl}^{-}$channel, hyperpolarizing the cell membrane, preventing the influx of $\mathrm{Ca}^{2+}$, and inhibiting the effect of $\mathrm{Ca}^{2+}$ on the growth and migration of endothelial cells (38); (2) Reducing the oxidation of nitric oxide through a glutathione-dependent mechanism, thereby increasing its content in the circulatory system (39); (3) Increasing the synthesis of glutathione in vascular inherent cells, thus playing a cytoprotective role (40).

Lack of Gly or mutations in the coding gene may result in decreased synthesis of elastin and collagen or abnormal structure, by which the blood vessel wall will become weak and gradually expand to form aneurysms under the pressure of blood flow (41). Moreover, studies have reported that glycine inhibits the production of reactive oxygen species (ROS) by synthesizing GSH to reduce the oxidative stress response of the blood vessel wall (42), and Gly can also regulate glucose and lipid metabolism $(35,43,44)$. These findings suggest that glycine is involved in the pathophysiological progress of AAA, and further research is needed to study the mechanism (Figure 1d).

\section{GLYCOMETABOLISM AND AAA}

Glycometabolism refers to a series of complex chemical reactions of carbohydrates such as glucose and glycogen in vivo. Diabetes mellitus (DM) is a group of metabolic diseases characterized by hyperglycemia. Although DM is an important risk factor for cardiovascular events and atherosclerosis-related diseases (4547), epidemiological investigations have found that DM and fasting blood glucose is negatively related to AAA (48-50).

Miyama et al. (51) found that compared with normal blood glucose, AAA mice with hyperglycemia had less blood vessel expansion, arterial wall inflammatory cell infiltration, elastic fiber degradation and neoangiogenesis. Furthermore, the degree of AAA expansion and pathophysiological changes in diabetic mice treated with insulin were greater than those of diabetic AAA mice not treated with insulin, suggesting that hyperglycemia can limit the development of experimental aortic aneurysms. The mechanism may be linked to the down-regulation of vascular endothelial growth factor expression via interfering with the hypoxia-inducible factor-1 signaling pathway by hyperglycemia. Dua et al. (52) also found that hyperglycemia may also increase the level of endogenous plasminogen activator inhibitor1 , inhibit plasmin production, and reduce the infiltration of macrophages and the expression of MMPs in the arterial wall, thereby limiting the progress of experimental AAA.

Besides, chronic hyperglycemia can make extracellular matrix (ECM) glycation to form advanced glycation end-products (AGEs), which increases the stiffness of blood vessels (53). Koole et al. (54) found that the concentration of pentosidine, one of the AGEs, was negatively correlated with the diameter of the abdominal aorta in AAA patients with DM. The glycosylated AAA tissues can resist MMPs-induced degradation of type I collagen. Golledge et al. (55) incubated activated monocytes with glycosylated collagen fibers and found that glycosylated collagen tissue can inhibit monocytes from secreting MMPs. These findings indicate that AGEs induced by hyperglycemia may play a protective role in the progress of AAA (Figure 1e).

Clinical studies have also found that DM patients have a lower incidence of AAA (56), and AAA patients with DM have smaller aneurysms, lower expansion rate and are less likely to rupture $(57,58)$. In addition, some researchers used 
positron emission tomography/computed tomography to detect AAA tissue and found an increase in the uptake of $18 \mathrm{~F}$ fluorodeoxyglucose mediated by glucose transporters, suggesting enhanced glucose metabolism activity in the lesion tissue (59). Tsuruda et al. (60) found in mouse models that the enhancement of glycolytic activity in arterial wall tissue is one of the reasons for the development of AAA. They tried intraperitoneal administration of glycolysis inhibitor 2-deoxyglucose, a glucose analog and found that it can alleviate AAA via inhibiting macrophage survival and adhesion to endothelial cells, ECM degradation and inflammation. Later studies confirmed that the energy metabolism in macrophages changed from oxidative phosphorylation to glycolysis in the HHcy state, which promoted the production of pro-inflammatory cytokines and ROS, thereby aggravating AAA (61). These results suggest that interference with glycolytic activity may be a potential therapeutic target for AAA.

\section{LIPID METABOLISM AND AAA}

Lipids, produced by the dehydration condensation of fatty acids and alcohols, refer to ester compounds and their derivatives, including triglycerides (TG) and lipoid (phospholipids, glycolipids, sterols, and their esters). Atherosclerosis (AS) and AAA have some similar pathophysiological processes, such as chronic inflammation, VSMC apoptosis and phenotypic transformation. Although it is still uncertain whether AS and AAA have a causal relationship (62), some traditional risk factors for AS (such as hyperlipemia, hypertension, smoking, age, gender, etc.) are also related to AAA (63-67). Investigations and studies have found that serum low density lipoprotein cholesterol (LDL-C), total cholesterol (TC), and TG levels are positively correlated with AAA (64-66). Nevertheless, whether elevated TG level will promote the occurrence and development of AAA is still controversial $(68,69)$.

Cholesterol is often combined with lipoproteins in plasma, and low density lipoprotein (LDL) is the main carrier for transporting endogenous cholesterol. When TC and/or LDL$\mathrm{C}$ abnormally elevated beyond the normal range, it will lead to hypercholesterolemia. Hobbs et al. (70) found through casecontrol analysis that there is a significant positive correlation between LDL-C and AAA, and hypothesized that LDL-C may cause AAA by inducing chronic inflammation-mediated degradation of ECM. Weng et al. (71) confirmed the causal relationship between high serum TC and LDL-C levels and AAA through Mendelian randomization study. Liu et al. (72) confirmed that hypercholesterolemia can promote the occurrence and development of AAA in mice. However, there is still a lack of basic research to clarify the specific cellular and molecular mechanisms of hypercholesterolemia on AAA (Figure 2f).

Contrary to LDL, high density lipoprotein (HDL) is mainly responsible for the reverse transport of cholesterol. HDL transports extrahepatic cholesterol to the liver, where cholesterol is transformed into bile acids and excreted, so that the high density lipoprotein cholesterol (HDL-C) level of hyperlipidemia is reduced. There is sufficient evidence to prove that HDL-C level is negatively correlated with $\operatorname{AAA}(73,74)$, and the impaired cholesterol efflux caused by abnormal HDL transport function is also related to the development of $\operatorname{AAA}(75,76)$. A recent study found that IgG anti-HDL antibody levels in AAA patients were elevated, and the antibody levels were positively correlated with the aortic diameter and negatively correlated with HDL-C levels, suggesting that AAA patients may have humoral immune response against $\mathrm{HDL}$, which provides a new direction for the study of AAA pathogenesis and drug targets (77).

In addition, there is lipoprotein(a), also known as LP(a), in plasma which is related to AAA. $\operatorname{LP}(a)$ is a type of independent lipoprotein, which is produced by the liver and is not transformed into other lipoproteins, and its physiological function is currently unclear (78). Studies have shown that elevated LP(a) level is a risk factor for cardiovascular diseases. And current research is mainly about AS and thrombotic vascular diseases (79-82). Some researchers have also found that LP(a) levels are elevated in AAA patients (19). High plasma level of LP(a) may also be a risk factor for AAA (83), because LP(a) carries monocyte chemoattractant protein 1 and oxidized phospholipids, which can cause chronic inflammation and oxidative stress in the blood vessel wall (84-86), but the specific mechanism has not been confirmed (Figure $2 \mathrm{~g}$ ).

In recent years, it has been discovered that long chain polyunsaturated fatty acids (LCPUFAs) may be related to AAA. Arachidonic acid (ARA), a long chain omega-6 polyunsaturated fatty acid (LC n-6 PUFA), can be metabolized to produce prostaglandin E2, thromboxane A2, and leukotriene B4, which have been shown to aggravate AAA through their proinflammatory effect (87-90). The selective cyclooxygenase-2 (COX-2) inhibitor Celecoxib inhibits the formation of AAA in mice infused with AngII, which also confirms that the prostaglandin compounds produced by COX-2 catalyzed ARA play a certain role in AAA (91). Consistently, a clinical study conducted in Danish men showed that increased levels of ARA is related to AAA incidence and progression. AAA patients with high ARA levels were more likely to require surgical repair (92). Furthermore, this clinical study also found that the levels of eicosapentaenoic acid (EPA), a long chain omega-3 polyunsaturated fatty acid (LC n-3 PUFA), was not associated with AAA.

However, another Japanese clinical study found that EPA levels in AAA patients were relatively low compared with that in healthy person. There is a significant negative correlation between EPA levels or the EPA/arachidonic acid (ARA) ratio and AAA growth rate or maximum aneurysm diameter (93). A number of studies have found that LC n-3 PUFAs and their derivatives resolvins produced by enzymatic oxidation can inhibit the infiltration of inflammatory cells, the production of cytokines, the expression of MMPs, and oxidative stress (94-96). Moreover, LC n-3 PUFAs can reduce the synthesis of endogenous cholesterol and increase the metabolism of exogenous cholesterol, thereby reducing plasma TC level (9799). In vivo experiments have confirmed that LC n-3 PUFAs and their derivatives can inhibit the occurrence and development of AAA (Figure 2h) (94, 100, 101). The contradiction between two 


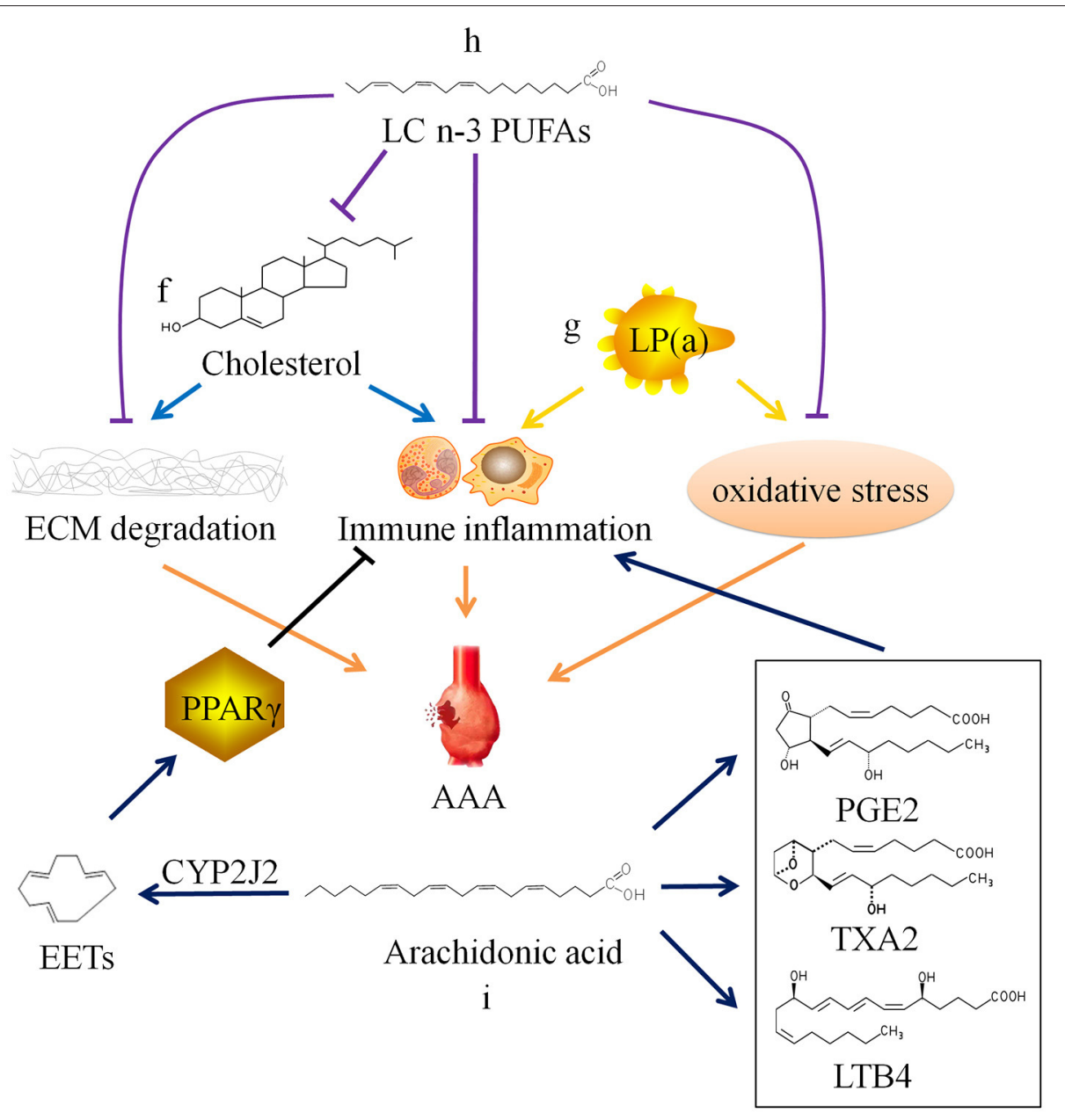

FIGURE 2 | Relationship between lipid metabolism and abdominal aortic aneurysm. ECM, extracellular matrix; AAA, abdominal aortic aneurysm; SMC, smooth muscle cell; LP(a), lipoprotein(a); LC n-3 PUFAs, long chain omega-3 polyunsaturated fatty acids; PGE2, prostaglandin E2; TXA2, thromboxane A2; LTB4, leukotriene B4; CYP2J2, Cytochrome P450 epoxygenase 2J2; EETs, epoxyeicosatrienoic acids; PPAR $\gamma$, peroxisome proliferator-activated receptor $\gamma$.

clinical studies results from a huge difference on EPA diet of the populations. Furthermore, Cytochrome P450 cyclooxygenase 2J2 (CYP2J2) catalyzes the formation of epoxyeicosatrienoic acids (EETs) from ARA. Cai et al. (102) found that increased levels of EETs through CYP2J2 overexpression can activate peroxisome proliferator-activated receptor $\gamma$ to exert anti-inflammatory effect, thereby preventing the development of AAA in mice (Figure 2i). These findings provide new ideas for the therapy of AAA.

Based on previous studies on the relationship between lipid metabolism and AAA, researchers are working on the development of preventive and therapeutic drugs for AAA from the perspective of regulating lipid metabolism. At present, the most widely used drug to regulate lipid metabolism is statins, the mechanism of which is to inhibit the rate-limiting enzyme 3hydroxy-3-methylglutaryl coenzyme A reductase in endogenous cholesterol synthesis, thereby effectively reducing TC and LDLC (103-105).

Moreover, proprotein convertase subtilisin/Kexin type 9 (PCSK9) inhibitors can reduce LDL-C by inhibiting the degradation of low density lipoprotein receptor $(L d l r)$, while PCSK9 gain-of-function mutation promotes AAA occurrence in mice (106). Previous clinical study has reported that PCSK9 inhibitors can reduce the risk of AAA (107). Evolocumab and Alirocumab, as PCSK9 inhibitors, have been approved for the clinical treatment of hyperlipidemia in many countries and regions $(108,109)$, but their actual effects on AAA have not been tested in clinical studies.

\section{CONCLUSIONS AND PERSPECTIVES}

Due to the concealment in the early stage and the lack of prevention and treatment methods, AAA is still a cardiovascular disease with high risk of death. Improving the diagnosis rate and cure rate, delaying the progression of AAA to prevent its rupture, and improving the prognosis are the goals that researchers and clinicians have been working on.

The above studies have found that the increase in plasma metabolites levels caused by abnormal amino acid and lipid metabolism is related to AAA. Therefore, clinicians can assess 
the risk of AAA formation by detecting the corresponding metabolites levels in plasma. For the elderly with a high incidence of AAA, clinicians can also provide dietary recommendations to achieve the purpose of early prevention. For patients who are diagnosed with AAA but have not yet met the surgical criteria or cannot tolerate surgery, drug therapy is still an important intervention (110). Although there is still a lack of recognized drugs that can treat AAA, experimental animal studies provide many new clues for potential drug targets.

In summary, under the general trend of multi-disciplinary cooperation, which leads to a number of new interdisciplinary sciences, it is in line with the trend of scientific development to study the pathogenesis and to develop therapeutic methods of AAA from a multi-disciplinary perspective. Moreover, with the rapid development of metabolomics in recent years, metabolomics methods have penetrated into many fields of medical science, including disease diagnosis and drug development. If the cellular and molecular mechanisms involved in the occurrence and development of AAA can be further elucidated from the perspective of metabolism, it will surely promote the application of metabolomics in the field of cardiovascular diseases. More specifically, it may provide new

\section{REFERENCES}

1. Nordon IM, Hinchliffe RJ, Loftus IM, Thompson MM. Pathophysiology and epidemiology of abdominal aortic aneurysms. Nat. Rev. Cardiol. (2011) 8:92-102. doi: 10.1038/nrcardio.2010.180

2. Sakalihasan N, Michel JB, Katsargyris A, Kuivaniemi H, Defraigne JO, Nchimi A, et al. Abdominal aortic aneurysms. Nat. Rev. Dis. Primers. (2018) 4:34. doi: 10.1038/s41572-018-0030-7

3. Basnyat PS, Biffin AH, Moseley LG, Hedges AR, Lewis MH. Mortality from ruptured abdominal aortic aneurysm in Wales. Br. J. Surg. (1999) 86:765-70. doi: 10.1046/j.1365-2168.1999.01170.x

4. Hinchliffe RJ, Bruijstens L, MacSweeney ST, Braithwaite BD. A randomised trial of endovascular and open surgery for ruptured abdominal aortic aneurysm - results of a pilot study and lessons learned for future studies. Eur. J. Vasc. Endovasc. Surg. (2006) 32:506-13. doi: 10.1016/j.ejvs.2006.05.016

5. Zhang J, Schmidt J, Ryschich E, Schumacher H, Allenberg JR. Increased apoptosis and decreased density of medial smooth muscle cells in human abdominal aortic aneurysms. Chin. Med. J. (2003) 116:1549-52.

6. Ganguly P, Alam SF. Role of homocysteine in the development of cardiovascular disease. Nutr. J. (2015) 14:6. doi: 10.1186/1475-2891-14-6

7. Hannibal L, Blom HJ. Homocysteine and disease: causal associations or epiphenomenons? Mol. Aspects Med. (2017) 53:36-42. doi: 10.1016/j.mam.2016.11.003

8. Wang WM, Jin HZ. Homocysteine: a Potential common route for cardiovascular risk and dna methylation in psoriasis. Chin. Med. J. (2017) 130:1980-6. doi: 10.4103/0366-6999.211895

9. Hankey GJ, Eikelboom JW. Homocysteine and vascular disease. Lancet. (1999) 354:407-13. doi: 10.1016/S0140-6736(98)11058-9

10. Yap S, Boers GH, Wilcken B, Wilcken DE, Brenton DP, Lee PJ, et al. Vascular outcome in patients with homocystinuria due to cystathionine beta-synthase deficiency treated chronically: a multicenter observational study. Arterioscler. Thromb. Vasc. Biol. (2001) 21:2080-5. doi: $10.1161 /$ hq1201.100225

11. Bublil EM, Majtan T. Classical homocystinuria: from cystathionine betasynthase deficiency to novel enzyme therapies. Biochimie. (2020) 173:48-56. doi: 10.1016/j.biochi.2019.12.007

12. Majtan T, Park I, Cox A, Branchford BR, di Paola J, Bublil EM, et al. Behavior, body composition, and vascular phenotype of homocystinuric ideas and methods for early prevention, progression retardation and prognosis improvement of AAA.

\section{AUTHOR CONTRIBUTIONS}

YH wrote this review. WGu, TF, and RG modified and supplemented the content of the review. $\mathrm{BL}$ and $\mathrm{WGe}$ adjusted the grammar and structure of the article. JW advised on the article writing and reviewed the final version. All authors contributed to the article and approved the submitted version.

\section{FUNDING}

This work was financially supported by the Chinese Academy of Medical Sciences Innovation Fund for Medical Sciences (Grant No. 2017-I2M-1-008), the National Natural Science Foundation of China (Grant Nos. 81622008 and 91739107), the Thousand Young Talents Program of China, and the Open Research Funds of the State Key Laboratory of Genetic Engineering (Grant No. SKLGE-1607). mice on methionine-restricted diet or enzyme replacement therapy. FASEB J. (2019) 33:12477-86. doi: 10.1096/fj.201901203R

13. Majtan T, Hulková H, Park I, Krijt J, KoŽich V, Bublil EM, et al. Enzyme replacement prevents neonatal death, liver damage, and osteoporosis in murine homocystinuria. FASEB J. (2017) 31:5495-506. doi: 10.1096/fj.201700565R

14. Tsarouhas K, Tsitsimpikou C, Apostolakis S, Haliassos A, Tzardi M, Panagiotou M, et al. Homocysteine and metalloprotease-3 and-9 in patients with ascending aorta aneurysms. Thromb Res. (2011) 128:e95-9. doi: 10.1016/j.thromres.2011.07.008

15. Lee SJ, Lee YS, Seo KW, Bae JU, Kim GH, Park SY, et al. Homocysteine enhances MMP-9 production in murine macrophages via ERK and Akt signaling pathways. Toxicol. Appl. Pharmacol. (2012) 260:89-94. doi: 10.1016/j.taap.2012.01.026

16. Siennicka A, Zuchowski M, Chełstowski K, Cnotliwy M, Clark JS, Jastrzebska M. Homocysteine-enhanced proteolytic and fibrinolytic processes in thin intraluminal thrombus and adjacent wall of abdominal aortic aneurysm: study in vitro. Biomed. Res. Int. (2018) 2018:3205324. doi: 10.1155/2018/3205324

17. Fan Y. Role of methionine-induced hyperhomocysteinemia in the pathogenesis of abdominal aortic aneurysm and its molecular mechanisms (Doctoral thesis). China Medical University, Taichung, Taiwan (2019).

18. Moroz P, Le MT, Norman PE. Homocysteine and abdominal aortic aneurysms. ANZ J. Surg. (2007) 77:329-32. doi: 10.1111/j.1445-2197.2007.04052.x

19. Sofi F, Marcucci R, Giusti B, Pratesi G, Lari B, Sestini I, et al. High levels of homocysteine, lipoprotein (a) and plasminogen activator inhibitor-1 are present in patients with abdominal aortic aneurysm. Thromb. Haemost. (2005) 94:1094-8. doi: 10.1160/TH0506-0431

20. Halazun KJ, Bofkin KA, Asthana S, Evans C, Henderson M, Spark JI. Hyperhomocysteinaemia is associated with the rate of abdominal aortic aneurysm expansion. Eur. J. Vasc. Endovasc. Surg. (2007) 33:391-4. doi: 10.1016/j.ejvs.2006.10.022

21. Wong YY, Golledge J, Flicker L, McCaul KA, Hankey GJ, van Bockxmeer FM, et al. Plasma total homocysteine is associated with abdominal aortic aneurysm and aortic diameter in older men. J. Vasc. Surg. (2013) 58:364-70. doi: $10.1016 /$ j.jvs.2013.01.046 
22. Cao H, Hu X, Zhang Q, Li J, Wang J, Shao Y, et al. Homocysteine level and risk of abdominal aortic aneurysm: a meta-analysis. PLoS ONE. (2014) 9:e85831. doi: 10.1371/journal.pone.0085831

23. Fan Y, Li N, Liu C, Dong H, Hu X. Excessive methionine supplementation exacerbates the development of abdominal aortic aneurysm in rats. J. Vasc. Res. (2019) 56:230-40. doi: 10.1159/000501313

24. Warsi AA, Davies B, Morris-Stiff G, Hullin D, Lewis MH. Abdominal aortic aneurysm and its correlation to plasma homocysteine, and vitamins. Eur. J. Vasc. Endovasc. Surg. (2004) 27:75-9. doi: 10.1016/j.ejvs.2003.09.001

25. Wang Q, Ding Y, Song P, Zhu H, Okon I, Ding YN, et al. Tryptophan-derived 3-hydroxyanthranilic acid contributes to angiotensin II-induced abdominal aortic aneurysm formation in mice in vivo. Circulation. (2017) 136:2271-83. doi: 10.1161/CIRCULATIONAHA.117.030972

26. Rongvaux A, Andris F, Van Gool F, Leo O. Reconstructing eukaryotic NAD metabolism. Bioessays. (2003) 25:683-90. doi: 10.1002/bies.10297

27. Metghalchi S, Vandestienne M, Haddad Y, Esposito B, Dairou J, Tedgui A, et al. Indoleamine 2 3-dioxygenase knockout limits angiotensin II-induced aneurysm in low density lipoprotein receptor-deficient mice fed with high fat diet. PLoS ONE. (2018) 13:e0193737. doi: 10.1371/journal.pone.0193737

28. Prendergast GC, Malachowski WJ, Mondal A, Scherle P, Muller AJ. Indoleamine 2,3-dioxygenase and its therapeutic inhibition in cancer. Int. Rev. Cell Mol. Biol. (2018) 336:175-203. doi: 10.1016/bs.ircmb.2017. 07.004

29. Kim HW, Blomkalns AL, Ogbi M, Thomas M, Gavrila D, Neltner BS, et al. Role of myeloperoxidase in abdominal aortic aneurysm formation: mitigation by taurine. Am. J. Physiol. Heart Circ. Physiol. (2017) 313: H116879. doi: 10.1152/ajpheart.00296.2017

30. Webb NR, De Beer MC, Wroblewski JM, Ji A, Bailey W, Shridas $\mathrm{P}$, et al. Deficiency of endogenous acute-phase serum amyloid A protects apoE-/- mice from angiotensin II-induced abdominal aortic aneurysm formation. Arterioscler. Thromb. Vasc. Biol. (2015) 35:1156-65. doi: 10.1161/ATVBAHA.114.304776

31. Qin Y, Wang Y, Liu O, Jia L, Fang W, Du J, et al. Tauroursodeoxycholic acid attenuates angiotensin II induced abdominal aortic aneurysm formation in apolipoprotein E-deficient mice by inhibiting endoplasmic reticulum stress. Eur. J. Vasc. Endovasc. Surg. (2017) 53:337-45. doi: 10.1016/j.ejvs.2016.10.026

32. Petrat F, Boengler K, Schulz R, de Groot H. Glycine, a simple physiological compound protecting by yet puzzling mechanism(s) against ischaemiareperfusion injury: current knowledge. Br. J. Pharmacol. (2012) 165:2059-72. doi: 10.1111/j.1476-5381.2011.01711.x

33. Zhong X, Li X, Qian L, Xu Y, Lu Y, Zhang J, et al. Glycine attenuates myocardial ischemia-reperfusion injury by inhibiting myocardial apoptosis in rats. J. Biomed. Res. (2012) 26:346-54. doi: 10.7555/JBR.26.20110124

34. Díaz-Flores M, Cruz M, Duran-Reyes G, Munguia-Miranda C, LozaRodríguez H, Pulido-Casas E, et al. Oral supplementation with glycine reduces oxidative stress in patients with metabolic syndrome, improving their systolic blood pressure. Can. J. Physiol. Pharmacol. (2013) 91:855-60. doi: 10.1139/cjpp-2012-0341

35. El Hafidi M, Pérez I, Zamora J, Soto V, Carvajal-Sandoval G, Baños G. Glycine intake decreases plasma free fatty acids, adipose cell size, and blood pressure in sucrose-fed rats. Am. J. Physiol. Regul. Integr. Comp. Physiol. (2004) 287:R1387-93. doi: 10.1152/ajpregu.00159.2004

36. Wang W, Wu Z, Dai Z, Yang Y, Wang J, Wu G. Glycine metabolism in animals and humans: implications for nutrition and health. Amino Acids. (2013) 45:463-77. doi: 10.1007/s00726-013-1493-1

37. Cieslik KA, Sekhar RV, Granillo A, Reddy A, Medrano G, Heredia $\mathrm{CP}$, et al. Improved cardiovascular function in old mice after $\mathrm{N}$ acetyl cysteine and glycine supplemented diet: inflammation and mitochondrial factors. J. Gerontol. A Biol. Sci. Med. Sci. (2018) 73:1167-77. doi: 10.1093/gerona/gly034

38. Yamashina S, Konno A, Wheeler MD, Rusyn I, Rusyn EV, Cox AD, et al. Endothelial cells contain a glycine-gated chloride channel. Nutr. Cancer. (2001) 40:197-204. doi: 10.1207/S15327914NC402_17

39. $\mathrm{Wu} \mathrm{G}$, Meininger CJ. Regulation of nitric oxide synthesis by dietary factors. Annu. Rev. Nutr. (2002) 22:61-86. doi: 10.1146/annurev.nutr.22.110901.145329
40. Wu G, Fang YZ, Yang S, Lupton JR, Turner ND. Glutathione metabolism and its implications for health. J. Nutr. (2004) 134:489-92. doi: $10.1093 /$ jn/134.3.489

41. Anderson DW, Edwards TK, Ricketts MH, Kuivaniemi H, Tromp G, Stolle $\mathrm{CA}$, et al. Multiple defects in type III collagen synthesis are associated with the pathogenesis of abdominal aortic aneurysms. Ann. N.Y. Acad. Sci. (1996) 800:216-28. doi: 10.1111/j.1749-6632.1996.tb33312.x

42. Ruiz-Ramírez A, Ortiz-Balderas E, Cardozo-Saldaña G, Diaz-Diaz E, ElHafidi M. Glycine restores glutathione and protects against oxidative stress in vascular tissue from sucrose-fed rats. Clin. Sci. (2014) 126:19-29. doi: 10.1042/CS20130164

43. Pérez-Torres I, Ibarra B, Soria-Castro E, Torrico-Lavayen R, Pavón N, DiazDiaz E, et al. Effect of glycine on the cyclooxygenase pathway of the kidney arachidonic acid metabolism in a rat model of metabolic syndrome. Can. J. Physiol. Pharmacol. (2011) 89:899-910. doi: 10.1139/y11-086

44. Thalacker-Mercer AE, Ingram KH, Guo F, Ilkayeva O, Newgard CB, Garvey WT. BMI, RQ, diabetes, and sex affect the relationships between amino acids and clamp measures of insulin action in humans. Diabetes. (2014) 63:791-800. doi: 10.2337/db13-0396

45. de Ferranti SD, de Boer IH, Fonseca V, Fox CS, Golden SH, Lavie CJ, et al. Type 1 diabetes mellitus and cardiovascular disease: a scientific statement from the American Heart Association and American Diabetes Association. Circulation. (2014) 130:1110-30. doi: 10.1161/CIR.0000000000000034

46. Newman JD, Schwartzbard AZ, Weintraub HS, Goldberg IJ, Berger JS. Primary prevention of cardiovascular disease in diabetes mellitus. J. Am. Coll. Cardiol. (2017) 70:883-93. doi: 10.1016/j.jacc.2017.07.001

47. Shah AD, Langenberg C, Rapsomaniki E, Denaxas S, Pujades-Rodriguez M, Gale CP, et al. Type 2 diabetes and incidence of cardiovascular diseases: a cohort study in 1.9 million people. Lancet Diabetes Endocrinol. (2015) 3:105-13. doi: 10.1016/S2213-8587(14)70219-0

48. Kubota Y, Folsom AR, Pankow JS, Wagenknecht LE, Tang W. Diabetesrelated factors and abdominal aortic aneurysm events: the atherosclerotic risk in communities study. Ann. Epidemiol. (2018) 28:102-6.e101. doi: 10.1016/j.annepidem.2017.12.003

49. De Rango P, Farchioni L, Fiorucci B, Lenti M. Diabetes and abdominal aortic aneurysms. Eur. J. Vasc. Endovasc. Surg. (2014) 47:243-61. doi: 10.1016/j.ejvs.2013.12.007

50. Takagi H, Umemoto $T$. Negative association of diabetes with rupture of abdominal aortic aneurysm. Diab. Vasc. Dis. Res. (2016) 13:341-7. doi: $10.1177 / 1479164116651389$

51. Miyama N, Dua MM, Yeung JJ, Schultz GM, Asagami T, Sho E, et al. Hyperglycemia limits experimental aortic aneurysm progression. J. Vasc. Surg. (2010) 52:975-83. doi: 10.1016/j.jvs.2010.05.086

52. Dua MM, Miyama N, Azuma J, Schultz GM, Sho M, Morser J, et al. Hyperglycemia modulates plasminogen activator inhibitor-1 expression and aortic diameter in experimental aortic aneurysm disease. Surgery. (2010) 148:429-35. doi: 10.1016/j.surg.2010.05.014

53. Aronson D. Cross-linking of glycated collagen in the pathogenesis of arterial and myocardial stiffening of aging and diabetes. J. Hypertens. (2003) 21:3-12. doi: 10.1097/00004872-200301000-00002

54. Koole D, van Herwaarden JA, Schalkwijk CG, Lafeber F, Vink A, Smeets $\mathrm{MB}$, et al. A potential role for glycated cross-links in abdominal aortic aneurysm disease. J. Vasc. Surg. (2017) 65:1493-503.e1493. doi: 10.1016/j.jvs.2016.04.028

55. Golledge J, Karan M, Moran CS, Muller J, Clancy P, Dear AE, et al. Reduced expansion rate of abdominal aortic aneurysms in patients with diabetes may be related to aberrant monocyte-matrix interactions. Eur. Heart J. (2008) 29:665-72. doi: 10.1093/eurheartj/ehm557

56. Shantikumar S, Ajjan R, Porter KE, Scott DJ. Diabetes and the abdominal aortic aneurysm. Eur. J. Vasc. Endovasc. Surg. (2010) 39:200-7. doi: 10.1016/j.ejvs.2009.10.014

57. Lareyre F, Hassen-Khodja R, Raffort J. Translational applications of glucose metabolism in abdominal aortic aneurysm. J. Vasc. Surg. (2019) 70:2093-7. doi: 10.1016/j.jvs.2019.03.021

58. Raffort J, Lareyre F, Clément M, Hassen-Khodja R, Chinetti G, Mallat Z. Diabetes and aortic aneurysm: current state of the art. Cardiovasc. Res. (2018) 114:1702-13. doi: 10.1093/cvr/cvy174 
59. Kotze CW, Menezes LJ, Endozo R, Groves AM, Ell PJ, Yusuf SW. Increased metabolic activity in abdominal aortic aneurysm detected by $18 \mathrm{~F}$ fluorodeoxyglucose (18F-FDG) positron emission tomography/computed tomography (PET/CT). Eur. J. Vasc. Endovasc. Surg. (2009) 38:93-9. doi: 10.1016/j.ejvs.2008.12.016

60. Tsuruda T, Hatakeyama K, Nagamachi S, Sekita Y, Sakamoto S, Endo GJ, et al. Inhibition of development of abdominal aortic aneurysm by glycolysis restriction. Arterioscler. Thromb. Vasc. Biol. (2012) 32:1410-7. doi: 10.1161/ATVBAHA.111.237065

61. Sun W, Pang Y, Liu Z, Sun L, Liu B, Xu M, et al. Macrophage inflammasome mediates hyperhomocysteinemia-aggravated abdominal aortic aneurysm. J. Mol. Cell Cardiol. (2015) 81:96-106. doi: 10.1016/j.yjmcc.2015.02.005

62. Johnsen SH, Forsdahl SH, Singh K, Jacobsen BK. Atherosclerosis in abdominal aortic aneurysms: a causal event or a process running in parallel? The Tromsø study. Arterioscler. Thromb. Vasc. Biol. (2010) 30:1263-8. doi: 10.1161/ATVBAHA.110.203588

63. Toghill BJ, Saratzis A, Bown MJ. Abdominal aortic aneurysm-an independent disease to atherosclerosis? Cardiovasc. Pathol. (2017) 27:71-5. doi: 10.1016/j.carpath.2017.01.008

64. Wanhainen A, Bergqvist D, Boman K, Nilsson TK, Rutegård J, Björck M. Risk factors associated with abdominal aortic aneurysm: a populationbased study with historical and current data. J. Vasc. Surg. (2005) 41:390-6. doi: 10.1016/j.jvs.2005.01.002

65. Forsdahl SH, Singh K, Solberg S, Jacobsen BK. Risk factors for abdominal aortic aneurysms: a 7-year prospective study: the Tromsø Study, 1994-2001. Circulation. (2009) 119:2202-8. doi: 10.1161/CIRCULATIONAHA.108.817619

66. Iribarren C, Darbinian JA, Go AS, Fireman BH, Lee CD, Grey DP. Traditional and novel risk factors for clinically diagnosed abdominal aortic aneurysm: the Kaiser multiphasic health checkup cohort study. Ann. Epidemiol. (2007) 17:669-78. doi: 10.1016/j.annepidem.2007.02.004

67. Watt HC, Law MR, Wald NJ, Craig WY, Ledue TB, Haddow JE. Serum triglyceride: a possible risk factor for ruptured abdominal aortic aneurysm. Int. J. Epidemiol. (1998) 27:949-52. doi: 10.1093/ije/27.6.949

68. Moxon JV, Liu D, Wong G, Weir JM, Behl-Gilhotra R, Bradshaw B, et al. Comparison of the serum lipidome in patients with abdominal aortic aneurysm and peripheral artery disease. Circ. Cardiovasc. Genet. (2014) 7:71-9. doi: 10.1161/CIRCGENETICS.113.000343

69. Golledge J, van Bockxmeer F, Jamrozik K, McCann M, Norman PE. Association between serum lipoproteins and abdominal aortic aneurysm. Am. J. Cardiol. (2010) 105:1480-4. doi: 10.1016/j.amjcard.2009.12.076

70. Hobbs SD, Claridge MW, Quick CR, Day NE, Bradbury AW, Wilmink AB. LDL cholesterol is associated with small abdominal aortic aneurysms. Eur. J. Vasc. Endovasc. Surg. (2003) 26:618-22. doi: 10.1016/S1078-5884(03)00412-X

71. Weng LC, Roetker NS, Lutsey PL, Alonso A, Guan W, Pankow JS, et al. Evaluation of the relationship between plasma lipids and abdominal aortic aneurysm: a Mendelian randomization study. PLOS ONE. (2018) 13:e0195719. doi: 10.1371/journal.pone.0195719

72. Liu J, Sawada H, Howatt DA, Moorleghen JJ, Vsevolozhskaya O, Daugherty A, et al. Hypercholesterolemia accelerates both the initiation and progression of angiotensin II-induced abdominal aortic aneurysms. Ann. Vasc. Med. Res. (2020) 6:1099. doi: 10.1101/2020.01.03.893313

73. Takagi H, Manabe H, Kawai N, Goto SN, Umemoto T. Serum high-density and low-density lipoprotein cholesterol is associated with abdominal aortic aneurysm presence: a systematic review and meta-analysis. Int. Angiol. (2010) 29:371-5.

74. Harrison SC, Holmes MV, Burgess S, Asselbergs FW, Jones GT, Baas $\mathrm{AF}$, et al. Genetic association of lipids and lipid drug targets with abdominal aortic aneurysm: a meta-analysis. JAMA Cardiol. (2018) 3:26-33. doi: 10.1001/jamacardio.2017.4293

75. Martínez-López D, Cedó L, Metso J, Burillo E, García-León A, Canyelles M, et al. Impaired HDL (high-density lipoprotein)-mediated macrophage cholesterol efflux in patients with abdominal aortic aneurysm-brief report. Arterioscler. Thromb. Vasc. Biol. (2018) 38:2750-4. doi: 10.1161/ATVBAHA.118.311704

76. Mourmoura E, Vasilaki A, Giannoukas A, Michalodimitrakis E, Pavlidis P, Tsezou A. Evidence of deregulated cholesterol efflux in abdominal aortic aneurysm. Acta Histochem. (2016) 118:97-108. doi: 10.1016/j.acthis.2015.11.012

77. Rodríguez-Carrio J, Lindholt JS, Canyelles M, Martínez-López D, Tondo M, Blanco-Colio LM, et al. IgG anti-high density lipoprotein antibodies are elevated in abdominal aortic aneurysm and associated with lipid profile and clinical features. J. Clin. Med. (2019) 9:67. doi: 10.3390/jcm9010067

78. Schmidt K, Noureen A, Kronenberg F, Utermann G. Structure, function, and genetics of lipoprotein (a). J. Lipid Res. (2016) 57:1339-59. doi: 10.1194/jlr.R067314

79. Eckardstein AV. Lipoprotein(a). Eur. Heart J. (2017) 38:1530-2. doi: 10.1093/eurheartj/ehx233

80. Langsted A, Nordestgaard BG, Kamstrup PR. Elevated lipoprotein(a) and risk of ischemic stroke. J. Am. Coll. Cardiol. (2019) 74:54-66. doi: 10.1016/j.jacc.2019.03.524

81. Kamstrup PR, Tybjaerg-Hansen A, Steffensen R, Nordestgaard BG. Genetically elevated lipoprotein(a) and increased risk of myocardial infarction. JAMA. (2009) 301:2331-9. doi: 10.1001/jama.2009.801

82. Ma KL, Gong TK, Hu ZB, Zhang Y, Wang GH, Liu L, et al. Lipoprotein(a) accelerated the progression of atherosclerosis in patients with end-stage renal disease. BMC Nephrol. (2018) 19:192. doi: 10.1186/s12882-018-0986-2

83. Kotani K, Sahebkar A, Serban MC, Ursoniu S, Mikhailidis DP, Mariscalco $\mathrm{G}$, et al. Lipoprotein(a) levels in patients with abdominal aortic aneurysm. Angiology. (2017) 68:99-108. doi: 10.1177/0003319716637792

84. Wiesner P, Tafelmeier M, Chittka D, Choi SH, Zhang L, Byun YS, et al. MCP1 binds to oxidized LDL and is carried by lipoprotein(a) in human plasma. J. Lipid Res. (2013) 54:1877-83. doi: 10.1194/jlr.M036343

85. Leibundgut G, Scipione C, Yin H, Schneider M, Boffa MB, Green S, et al. Determinants of binding of oxidized phospholipids on apolipoprotein (a) and lipoprotein (a). J. Lipid Res. (2013) 54:2815-30. doi: 10.1194/jlr.M040733

86. Taleb A, Witztum JL, Tsimikas S. Oxidized phospholipids on apoB100-containing lipoproteins: a biomarker predicting cardiovascular disease and cardiovascular events. Biomark Med. (2011) 5:673-94. doi: $10.2217 / \mathrm{bmm} .11 .60$

87. Bayston T, Ramessur S, Reise J, Jones KG, Powell JT. Prostaglandin E2 receptors in abdominal aortic aneurysm and human aortic smooth muscle cells. J. Vasc. Surg. (2003) 38:354-9. doi: 10.1016/S0741-5214(03) 00339-2

88. Holmes DR, Wester W, Thompson RW, Reilly JM. Prostaglandin E2 synthesis and cyclooxygenase expression in abdominal aortic aneurysms. J. Vasc. Surg. (1997) 25:810-5. doi: 10.1016/S0741-5214(97)70210-6

89. Ahluwalia N, Lin AY, Tager AM, Pruitt IE, Anderson TJ, Kristo F, et al. Inhibited aortic aneurysm formation in BLT1-deficient mice. J. Immunol. (2007) 179:691-7. doi: 10.4049/jimmunol.179.1.691

90. Courtois A, Makrygiannis G, Cheramy-Bien JP, Purnelle A, Pirotte B, Dogne $\mathrm{JM}$, et al. Therapeutic applications of prostaglandins and thromboxane A2 inhibitors in abdominal aortic aneurysms. Curr. Drug Targets. (2018) 19:1247-55. doi: 10.2174/1389450119666171227224314

91. King VL, Trivedi DB, Gitlin JM, Loftin CD. Selective cyclooxygenase2 inhibition with celecoxib decreases angiotensin II-induced abdominal aortic aneurysm formation in mice. Arterioscler. Thromb. Vasc. Biol. (2006) 26:1137-43. doi: 10.1161/01.ATV.0000216119.79008.ac

92. Lindholt JS, Kristensen KL, Burillo E, Martinez-Lopez D, Calvo C, Ros E, et al. Arachidonic Acid, but not omega-3 index, relates to the prevalence and progression of abdominal aortic aneurysm in a populationbased study of Danish men. J. Am. Heart Assoc. (2018) 7:e007790. doi: 10.1161/JAHA.117.007790

93. Aikawa T, Miyazaki T, Shimada K, Sugita Y, Shimizu M, Ouchi S, et al. Low serum levels of EPA are associated with the size and growth rate of abdominal aortic aneurysm. J. Atheroscler. Thromb. (2017) 24:912-20. doi: $10.5551 /$ jat.38315

94. Meital LT, Sandow SL, Calder PC, Russell FD. Abdominal aortic aneurysm and omega-3 polyunsaturated fatty acids: mechanisms, animal models, and potential treatment. Prostaglandins Leukot Essent. Fatty Acids. (2017) 118:19. doi: 10.1016/j.plefa.2017.02.001

95. Meital LT, Windsor MT, Perissiou M, Schulze K, Magee R, Kuballa A, et al. Omega-3 fatty acids decrease oxidative stress and inflammation in macrophages from patients with small abdominal aortic aneurysm. Sci. Rep. (2019) 9:12978. doi: 10.1038/s41598-019-49362-Z 
96. Pope NH, Salmon M, Davis JP, Chatterjee A, Su G, Conte MS, et al. $\mathrm{D}$-series resolvins inhibit murine abdominal aortic aneurysm formation and increase M2 macrophage polarization. FASEB J. (2016) 30:4192-201. doi: 10.1096/fj.201600144RR

97. Oh Y, Jin Y, Park Y. Synergic hypocholesterolaemic effect of n-3 PUFA and oestrogen by modulation of hepatic cholesterol metabolism in female rats. Br. J. Nutr. (2015) 114:1766-73. doi: 10.1017/S0007114515003517

98. Yuan F, Wang H, Tian Y, Li Q, He L, Li N, et al. Fish oil alleviated highfat diet-induced non-alcoholic fatty liver disease via regulating hepatic lipids metabolism and metaflammation: a transcriptomic study. Lipids Health Dis. (2016) 15:20. doi: 10.1186/s12944-016-0190-y

99. Pizzini A, Lunger L, Demetz E, Hilbe R, Weiss G, Ebenbichler C, et al. The role of omega-3 fatty acids in reverse cholesterol transport: a review. Nutrients. (2017) 9:1099. doi: 10.3390/nu9101099

100. Akagi D, Hoshina K, Watanabe T, Conte MS. Drug therapy for abdominal aortic aneurysms utilizing omega-3 unsaturated fatty acids and their derivatives. Curr. Drug Targets. (2018) 19:1309-17. doi: 10.2174/1389450118666171013101815

101. Yoshihara T, Shimada K, Fukao K, Sai E, Sato-Okabayashi Y, Matsumori R, et al. Omega 3 polyunsaturated fatty acids suppress the development of aortic aneurysms through the inhibition of macrophage-mediated inflammation. Circ. J. (2015) 79:1470-8. doi: 10.1253/circj.CJ-14-0471

102. Cai Z, Zhao G, Yan J, Liu W, Feng W, Ma B, et al. CYP2J2 overexpression increases EETs and protects against angiotensin II-induced abdominal aortic aneurysm in mice. J. Lipid Res. (2013) 54:1448-56. doi: 10.1194/jlr.M036533

103. Chou R, Dana T, Blazina I, Daeges M, Jeanne TL. Statins for prevention of cardiovascular disease in adults: evidence report and systematic review for the US preventive services task force. JAMA. (2016) 316:2008-24. doi: 10.1001/jama.2015.15629

104. Adhyaru BB, Jacobson TA. Safety and efficacy of statin therapy. Nat. Rev. Cardiol. (2018) 15:757-69. doi: 10.1038/s41569-018-0098-5

105. Ye YC, Zhao XL, Zhang SY. Use of atorvastatin in lipid disorders and cardiovascular disease in Chinese patients. Chin. Med. J. (2015) 128:259-66. doi: 10.4103/0366-6999.149226
106. Lu H, Howatt DA, Balakrishnan A, Graham MJ, Mullick AE, Daugherty A. Hypercholesterolemia induced by a PCSK9 gainof-function mutation augments angiotensin II-induced abdominal aortic aneurysms in C57BL/6 mice-brief report. Arterioscler. Thromb. Vasc. Biol. (2016) 36:1753-7. doi: 10.1161/ATVBAHA.116. 307613

107. Klarin D, Damrauer SM, Cho K, Sun YV, Teslovich TM, Honerlaw J, et al Genetics of blood lipids among $\sim 300,000$ multi-ethnic participants of the Million Veteran Program. Nat. Genet. (2018) 50:1514-23. doi: 10.1038/s41588-018-0222-9

108. Sabatine MS, Giugliano RP, Keech AC, Honarpour N, Wiviott SD, Murphy SA, et al. Evolocumab and clinical outcomes in patients with cardiovascular disease. N. Engl. J. Med. (2017) 376:1713-22. doi: 10.1056/NEJMoa16 15664

109. Robinson JG, Farnier M, Krempf M, Bergeron J, Luc G, Averna M, et al. Efficacy and safety of alirocumab in reducing lipids and cardiovascular events. N. Engl. J. Med. (2015) 372:1489-99. doi: 10.1056/NEJMoa15 01031

110. Yoshimura K, Morikage N, Nishino-Fujimoto S, Furutani A, Shirasawa B, Hamano K. Current status and perspectives on pharmacologic therapy for abdominal aortic aneurysm. Curr. Drug Targets. (2018) 19:1265-75. doi: $10.2174 / 1389450119666171227223331$

Conflict of Interest: The authors declare that the research was conducted in the absence of any commercial or financial relationships that could be construed as a potential conflict of interest.

Copyright (c) $2021 \mathrm{Hou}, \mathrm{Guo}, \mathrm{Fan}, \mathrm{Li}, \mathrm{Ge}$, Gao and Wang. This is an open-access article distributed under the terms of the Creative Commons Attribution License (CC $B Y)$. The use, distribution or reproduction in other forums is permitted, provided the original author(s) and the copyright owner(s) are credited and that the original publication in this journal is cited, in accordance with accepted academic practice. No use, distribution or reproduction is permitted which does not comply with these terms. 